\title{
Understanding the Two Components of Risk Attitudes: An Experimental Analysis
}

\author{
Jianying Qiu \\ Department of Finance, University of Vienna, 1210 Vienna, Austria, jianying.qiu@univie.ac.at \\ Eva-Maria Steiger \\ Strategic Interaction Group, Max Planck Institute of Economics, 07745 Jena, Germany, steiger@econ.mpg.de
}

\begin{abstract}
C umulative prospect theory introduced the weighting of probabilities as an additional component to capture risk attitudes. However, this addition would be a less significant challenge to expected utility theory (EU) if utility curvature and probability weighting showed strong positive correlation. In that case the utility curvature in EU alone, although not properly describing risky behavior in general, would still capture most of the variance of individual risk aversion. This study provides experimental evidence that such a strong and positive correlation does not exist. Although most individuals exhibit concave utility and convex probability weighting, the two components show no strong positive correlation.
\end{abstract}

Key words: risk attitude; cumulative prospect theory; experimental study

History: Received April 20, 2009; accepted August 16, 2010, by Peter Wakker, decision analysis. Published online in Articles in Advance December 3, 2010.

\section{Introduction}

In expected utility theory (hereafter EU), the attitude toward risk originates from changes in marginal utility (i.e., the curvature of the utility function). As a result, risk attitudes are traditionally captured by fitting the best EU model and then using the individual utility curvature as the sole index of risk attitude. Kahneman and Tversky (1979) demonstrated various empirical deficiencies of that view. In (cumulative) prospect theory (hereafter PT) they added a second component to assess risk attitudes, namely the weighting of probabilities. Wakker (1994) gave an intuitive interpretation on the distinct roles that these two components play in the assessment of risk attitudes: utility describes an intrinsic appreciation of money prior to probability or risk, whereas risk attitude originates from the perception of probabilities.

Studies testing PT or measuring these two components of risk attitudes are abundant (see, e.g., Harrison and Rutström 2008 for a review). Yet to the best of our knowledge, no study has so far addressed the relation between these two components. Previous literature has either been silent on this relation or implicitly assumed independence. Addressing this question is, however, important, because PT would pose a less significant challenge to EU if the two components, utility curvature and probability weighting, were significantly and positively correlated (more concave in utility corresponds to more underweighting in probability). In that case, one could argue that the utility curvature in EU-although not properly describing risk behavior in general-would still capture most of the variance regarding degrees of risk aversion, making the other component of risk redundant.

In this study we investigate the relation between these two components of risk. Are they truly unconnected? Can an individual be risk seeking in one component and risk averse in the other? Results from our controlled laboratory experiment suggest that this may well be the case. Although most individuals in our study exhibit concave utility and convex probability weighting, we find no significant positive correlation between the two components. In a broader context, our results provide further evidence that measuring risk attitude through the curvature of utility alone may not be sufficient to describe decision making under risk, and that neglecting either of the two components entails a loss.

Because our aim is to understand the interplay of utility and probability weighting, a clean measurement of the two components is essential. In this study we employ the trade-off (TO) method (Wakker and Deneffe 1996) to measure risk attitude, which is the only method to date that allows for an independent measurement of the two components. Among others, Abdellaoui (2000), Abdellaoui et al. (2005), and van de Kuilen and Wakker (2011) have employed the TO method. In this paper we mostly rely on the TO method as introduced by Abdellaoui (2000). The rest of the paper is organized as follows: $\S 2$ outlines the procedure and experimental setup; technical details are in Appendices A and B. Section 3 contains the 
main results. The paper closes with a discussion and a conclusion in $\S \S 4$ and 5, respectively.

\section{The TO Method and Experimental Setup}

We restrict ourselves to PT for gains and binary lotteries with objective probabilities. Let $\left(x_{i+1}, p ; x_{i}\right)$ denote a prospect yielding $x_{i+1}$ with probability $p$ and $x_{i}$ otherwise. When $x_{i}<x_{i+1}$, this prospect is evaluated by $w(p) u\left(x_{i+1}\right)+[1-w(p)] u\left(x_{i}\right)$ in PT, where the utility function $u(\cdot)$ is assumed to be strictly increasing over the outcome space $[0, \infty)$, and the probability weighting function $w(\cdot)$ is increasing over the probability space $[0,1]$, with $w(0)=0$ and $w(1)=1$.

The TO method elicits utility and probability weights in two consecutive steps. In the first step (UT), a standard sequence of outcomes $x_{1}, \ldots, x_{n}$, i.e., equally spaced outcomes in terms of utility, is constructed. The second step (PW) uses this standard sequence to measure probability weights. More specifically, in UT an $x_{i+1}$ is determined to make the subject indifferent between $A:\left(x_{i+1}, p ; r\right)$ and $B:\left(x_{i}, p ; R\right)$, where $p, r$, and $R$ are exogenous parameters, and $x_{i}>R>r$. With $x_{i+1}$ at hand, $x_{i+2}$ is then varied in a similar way to make the subject indifferent between $A:\left(x_{i+2}, p ; r\right)$ and $B:\left(x_{i+1}, p ; R\right)$. According to PT, the two indifference relations imply

$$
\begin{aligned}
& {[1-w(p)] u(R)+w(p) u\left(x_{i}\right)} \\
& \quad=[1-w(p)] u(r)+w(p) u\left(x_{i+1}\right), \quad \text { and } \\
& \quad[1-w(p)] u(R)+w(p) u\left(x_{i+1}\right) \\
& \quad=[1-w(p)] u(r)+w(p) u\left(x_{i+2}\right), \\
& \quad \Rightarrow u\left(x_{i+2}\right)-u\left(x_{i+1}\right)=u\left(x_{i+1}\right)-u\left(x_{i}\right) .
\end{aligned}
$$

Combining the upper two equations leads to Equation (1), which states that the outcomes $\left(x_{i}, x_{i+1}, x_{i+2}\right)$ are equally spaced on the utility axis. Starting with a certain $x_{0}$ and constructing recursively $n$ times, we obtain a standard sequence of $x_{0}, x_{1}, \ldots, x_{n}$.

In PW, the obtained standard sequence of outcomes $x_{0}, x_{1}, \ldots, x_{n}$ is used to determine a sequence of probabilities. For each $x_{i}, i=1, \ldots, n-1$, a $p_{i}$ is determined to make the subject indifferent between a lottery $A:\left(x_{n}, p_{i} ; x_{0}\right)$ and a certain outcome $B:\left(x_{i}\right)$. According to PT the indifference implies

$$
\begin{aligned}
& w\left(p_{i}\right) u\left(x_{n}\right)+\left[1-w\left(p_{i}\right)\right] u\left(x_{0}\right)=w(1) u\left(x_{i}\right) \\
& \quad \Rightarrow w\left(p_{i}\right)=\frac{u\left(x_{i}\right)-u\left(x_{0}\right)}{u\left(x_{n}\right)-u\left(x_{0}\right)}, \quad \forall i=1, \ldots, n-1 .
\end{aligned}
$$

By (1), we know that $u\left(x_{i+1}\right)-u\left(x_{i}\right)$ is constant, and the above equation can be simplified in $w\left(p_{i}\right)=$ $i / n$, for $i=1, \ldots, n-1$. The elicited values of $p_{1}$, $p_{2}, \ldots, p_{n-1}$, along with the fact that $w\left(p_{i}\right)=i / n$, allow us to estimate the shape $w(p)$.
The experiment was conducted in June 2008 with 124 Jena University undergraduate students. ${ }^{1}$ We fixed the parameters at $p=0.5, r=0, R=10$, and $x_{0}=20$. We elicited six points for utility and five points for probabilities. Indifference was obtained by the modified bisection method, using eight iterations for each $x_{i}$, and seven iterations to obtain each $p_{i}{ }^{2}$ A consistency check for each $x_{i}$ was carried out by repeating the seventh choice. For probabilities we checked for consistency by eliciting a $p_{3}^{\prime}$ such that $\left(x_{3}\right) \sim\left(x_{4}, p_{3}^{\prime} ; x_{2}\right)$, which should equal to $p_{3}$ according to PT. This resulted in 54 rounds for the UT part and 42 rounds for the PW part. One round of each part was individually selected at random, the preferred lottery was played, and the resulting amount was paid privately and individually to the participant. The average earning was $€ 16$, with a min of $€ 8$ and max of $€ 46$. $^{3}$

\section{Results}

\subsection{Consistency and Reliability}

We repeated the seventh choice pair of each $x_{i}$ to check for consistency. Preference reversal occurred in $30 \%$ of the cases. This seemingly large number may originate from the small remaining interval for the inference of $x_{i}$ at the seventh choice ${ }^{4}$ and is comparable to the findings in Starmer and Sugden (1989) (26.5\%) and Camerer (1989) (31.6\%). In PW, we checked for consistency by comparing $\left(x_{6}, p_{3} ; x_{0}\right) \sim\left(x_{3}\right)$ and $\left(x_{4}, p_{3}^{\prime} ; x_{2}\right) \sim\left(x_{3}\right)$. According to PT, the two probabilities should be equal $\left(p_{3}=p_{3}^{\prime}\right)$. Indeed, the median values of $p_{3}$ and $p_{3}^{\prime}$ are equal to 0.5 , and they are not significantly different (paired Wilcoxon signed rank test $p>0.10) .^{5}$

\footnotetext{
${ }^{1}$ We ran four sessions, with 32 subjects in three sessions and 28 subjects in one session. Each session lasted about 90 minutes. The experiment included two further parts. Those results are reported in Qiu and Steiger (2010).

${ }^{2} \mathrm{~A}$ detailed description of the $\mathrm{TO}$ and the modified bisection method can be found in Appendix A. The procedure to elicit $p_{i}$ was made variable (seven or nine iterations) to accommodate inconsistent choices. However, this turned out to be unnecessary.

${ }^{3}$ We used z-Tree (Fischbacher 2007) for experimental software and ORSEE (Greiner 2004) to manage invitations to participants. An English translation of the original instructions can be found in Appendix B.

${ }^{4}$ Note that for $x_{1}$, when the interval is rather small (the difference was less than 2), preference reversal occurs in 39\% of the cases, while it lowers to $23 \%$ for $x_{6}$. This suggests that preference reversal is to some extent the result of small choice intervals. For larger intervals reversal is reduced yet still remains.

${ }^{5}$ The mean difference $p_{3}-p_{3}^{\prime}=-0.015$ and the mean and median absolute difference are 0.16 and 0.11 , respectively. The means are $p_{3}=0.6615$ and $p_{3}^{\prime}=0.6766$
} 
Table 1

Classification of Utility

\begin{tabular}{lccr}
\hline & \multicolumn{3}{c}{ Utility } \\
\cline { 2 - 4 } & $\tau_{u}$ & Difference & Both \\
\hline Concave & 71 & 67 & 59 \\
Convex & 27 & 9 & 6 \\
Linear & 26 & 25 & 12 \\
\hline
\end{tabular}

Note. The classification in the first column is according to the area ratio, then in the second column according to the nonparametric difference method, and finally in the third column those classified in the respective category according to both criteria.

\subsection{Classification of Utility Functions}

To classify each utility function, we calculated a ratio (denoted by $\tau_{u}$ ) of the area above the (linearly normalized) utility function and the rectangular area between $\left(x_{0}, 0\right)$ and $\left(x_{6}, 1\right){ }^{6}$ This ratio classified the concavity, convexity, or linearity of the utility function in a nonparametric way. For each subject, money was measured on the $x$-axis (from $x_{0}$ to $x_{6}$ ) and utility was measured on the $y$-axis (from 0 to 1 , at an increment of $\frac{1}{6}$ ). We calculated the area above these points (with linear approximation) and then normalized this measure by dividing the area by $1 \times\left(x_{6}-x_{0}\right)$. This gave a measure of $\tau_{u}$ between 0 and 1 , with risk aversion decreasing with the increase in $\tau_{u}$. We classified 26 subjects with a $\tau_{u}$ between $0.47 \leq \tau_{u} \leq 0.53$ as having linear utility functions. With this measure, 71 subjects had concave $\left(\tau_{u}<0.47\right)$ and 27 subjects had convex $\left(\tau_{u}>0.53\right)$ utility functions.

As a control, we also employed a nonparametric difference method to check for robustness of the above classification. Similar to Abdellaoui (2000), we took the first-order difference $\Delta_{i}^{\prime}=\left|x_{i}-x_{i-1}\right|$ for $i=$ $1, \ldots, 6$ and the second-order difference $\Delta_{j}^{\prime \prime}=\Delta_{j+1}^{\prime}-$ $\Delta_{j}^{\prime}$ for $j=1, \ldots, 5$. We classified 67 subjects as concave (with $\Delta_{j}^{\prime \prime}>2$ for three or more out of five times), 9 subjects as convex (with $\Delta_{j}^{\prime \prime}<-2$ for three or more out of five times), and 25 subjects as linear (with $-2 \leq \Delta_{i}^{\prime \prime} \leq 2$ for three or more out of five times). The remaining 23 subjects could not be classified with this method. As shown in Table 1, the majority of subjects was consistent with both classification methods, in particular those with concave utility functions. As a robustness check we also assumed a power form utility function $u(x)=x^{\alpha}$ and estimated an $\alpha$ for each individual with his or her sequence of values $x_{1}, x_{2}, \ldots, x_{6}$ using an ordinary least squares regression $\log (u(x))=$ Intercept $+\alpha \log (x)+\epsilon$, where $\epsilon \approx$ $N\left(0, \sigma^{2}\right)$. We found that the mean $\alpha$ equals 0.9316 and the median equals 0.8744 (standard error equals 0.0299), and that $74 \alpha$ s are significantly different

\footnotetext{
${ }^{6}$ Note that we calculated the area above, not below, the curve, i.e., the area between $u=1$ and the utility curve. This gives $\tau_{u}$ the same property as $\alpha$ of $u(x)=x^{\alpha}$ : an increase in $\tau_{u}$ and $\alpha$ both implies less risk aversion.
}

from 1 (at 5\% significant level), with $58 \alpha$ s significantly smaller than 1 , and $18 \alpha$ s significantly larger than 1 . The two measures $\left(\tau_{u}\right.$ and $\left.\alpha\right)$ are highly and positively correlated (Spearman's $\rho=0.6877$, $p<0.01$ ), which supports the use of $\tau_{u}$ as a measure of utility curvature.

\subsection{Classification of Probability Weighting Functions}

A classification of probability weighting requires careful consideration. Previous experiments found mostly inverse $S$-shaped, but also $S$-shaped, linear-, convex-, and concave-shaped probability weighting functions. First, we checked each subject's array of $p_{i}$ for patterns. The pattern of probability weighting is best discovered when $p$ is close to 0 or 1 , where probability weighting is supposed to be most pronounced. Thus, a simple way to detect patterns is to compare $w_{1}$ with $p_{1}$ and $w_{5}$ with $p_{5}$. We classified a probability weighting as convex (or optimistic) when $w_{1}<p_{1}$ and $w_{5}<p_{5}$, as concave (or pessimistic) when $w_{1}>p_{1}$ and $w_{5}>p_{5}$, as inverse $S$-shaped when $w_{1}>$ $p_{1}$ and $w_{5}<p_{5}$, and as $S$-shaped when $w_{1}<p_{1}$ and $w_{5}>p_{5}$. Based on these criteria, 83 subjects were convex, 4 subjects concave, 19 subjects inverse $S$-shaped, and 18 subjects $S$-shaped. Figure 1 gives a boxplot of the probability weights. The data pattern clearly favors a convex probability weighting.

Knowing the general pattern of probability weights, we took the area below the (linearly normalized) probability weights as a measure of pessimism. We used probabilities as $x$-axis (from $p_{1}$ to $p_{5}$ ) and decision weights as $y$-axis (from 0 to 1 , at an increment of $\frac{1}{6}$ ). We calculated the area below these points (with

Figure 1 Boxplot of the Probability Weighting

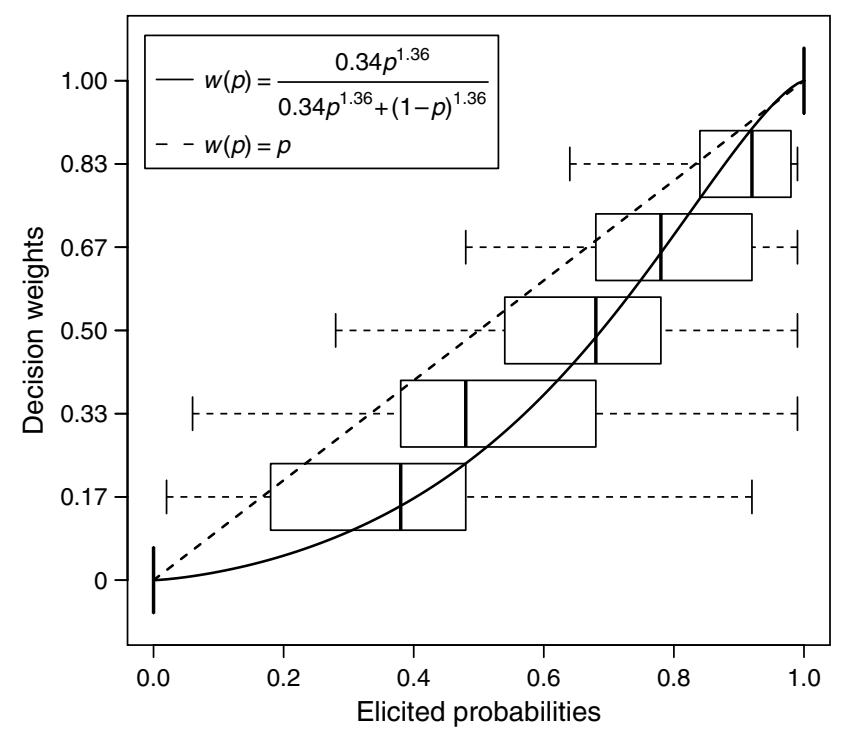

Note. The curve $w(p)=0.46 p^{1.08} /\left(0.46 p^{1.08}+(1-p)^{1.08}\right)$ is fitted with median data. 
Table 2

Classification of Probability Weighting

\begin{tabular}{lrlrr}
\hline \multicolumn{4}{c}{ Probability weighting } & \\
\hline & \multicolumn{1}{c}{$\tau_{p}$} & \multicolumn{2}{c}{ Nonparametric } & Both \\
\hline Convex/optimism & 97 & Convex & 83 & 77 \\
Concave/pessimism & 5 & Concave & 4 & 2 \\
Linear & 22 & S & 18 & - \\
& & Inverse $S$ & 19 & - \\
\hline
\end{tabular}

Note. Classification first according to the area ratio, then according to the nonparametric method, and finally those classified in the respective category according to both criteria.

linear approximation) to obtain a ratio (denoted by $\tau_{p}$ ) between 0 and 1 . The magnitude of $\tau_{p}$ states whether a subject, in general, overweights or underweights probabilities. To highlight the different components of risk attitudes, we classify subjects as pessimistic, neutral, and optimistic if their $\tau_{u}<0.47,0.47 \leq \tau_{u} \leq$ 0.53 , and $\tau_{u}>0.53$, respectively. With this measure, 97 subjects were pessimistic, 23 subjects were optimistic, and 33 subjects were neutral in probability weighting. Results and a comparison to the nonparametric method are shown in Table 2. Variations in the tolerance level did not alter results dramatically.

Because $\tau_{p}$ is merely an index of optimism or pessimism. It does not capture the degree of inverse $S$ or $S$. For robustness check, we also assumed a parametric form for the probability weighting function: $w(p)=\delta p^{\nu} /\left(\delta p^{\nu}+(1-p)^{\nu}\right)$. This form was introduced by Goldstein and Einhorn (1987) and has been frequently used. It allows for most common shapes of probability weighting, depending on the combination of $\delta$ and $\nu$, with $\delta$ being an index of pessimism and optimism. With five data points $p_{i}, i=1,2,3,4,5$, we estimated a $\delta$ and a $\nu$ for each subject by minimizing the sums of squared residuals. ${ }^{7}$ The median of $\delta$ equals 0.495 (mean $\delta=0.5679$, standard error equals 0.0316 ) and $\nu$ equals 0.88 (mean $\nu=1.0468$, standard error equals 0.0627). As we can see from Figure 1, the fitted curve using median data clearly favors a convex probability weighting function. The high correlation between $\tau_{p}$ and $\delta$ (two-sided Spearman's $\rho=0.8746$, $p<0.01$ ) also suggests that $\tau_{p}$ captures probability weighting reasonably well.

\subsection{Central Results}

Our main question, to which we now turn, is the relation between utility curvature and probability weights. The results are reported in Table 3 and Figure 2. The largest group in Table 3 are the subjects with concave utility functions and pessimism in the probability weighting (55 subjects). This finding is convenient for economists, because most theoretical

${ }^{7}$ We used a wide range of values, $\delta$ from 0 to 2 at an increment of $0.01, \nu$ from 0 to 4 at an increment of 0.04 .
Table 3 The Two Components of Risk Attitudes

\begin{tabular}{lcccr}
\hline & Concave $\tau_{u}$ & Linear $\tau_{u}$ & Convex $\tau_{u}$ & Sum \\
\hline Pessimistic $\tau_{p}$ & 55 & 16 & 26 & 97 \\
Neutral $\tau_{p}$ & 12 & 10 & 0 & 22 \\
Optimistic $\tau_{p}$ & 4 & 0 & 1 & 5 \\
Sum & 71 & 26 & 27 & 124 \\
\hline
\end{tabular}

Note. The columns depict the utility and the rows the probability weighting.

models rely on the assumption of risk averse agents. Our result suggests that the majority of the population may indeed be risk averse in both components (concave in utility and convex in probability weighting). There are further interesting patterns in the data. The third cell in the first row denotes the subjects with convex utility but pessimistic probability weighting. They are the second largest group in our classification ( 26 subjects). This is mirrored by the 4 subjects in the first cell of the third row. This cell denotes the subjects with concave utility but optimistic probability weighting.

In light of information obtained on the curvature of utility and probability weighting, a natural question is: Are subjects who are more concave in utility also more convex in probability weighting? To test this hypothesis, we ran a one-sided Spearman's $\rho$ rank correlation test of $\tau_{u}$ and $\tau_{p}$ for all subjects. It turned out that the correlation had the wrong sign and was not significant (Spearman's $\rho=-0.1597, p=0.9617$ )! The subjects with concave utility and convex probability weighting are those most often assumed in economic theories. As shown above, this is the largest

Figure 2 Distribution of the Area Ratios in Utility and Probability Weighting

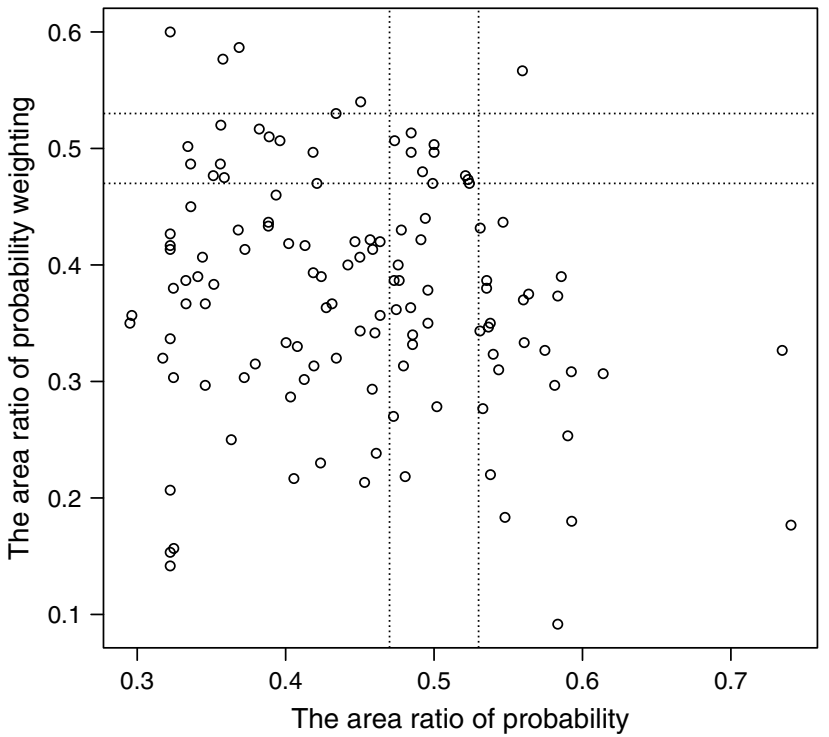

Notes. The lines are according to the classification of risk attitude. The largest group in the lower left field represents the subjects who are averse in utility and pessimistic in probability weighting. 
group in our data and most robust to different classification methods. However, for those subjects the correlation is not significant either (one-sided Spearman's rank correlation, $\rho=0.0701, p=0.6108$ ). Correlating the parametric estimator $\delta$ with $\alpha$ gives qualitatively the same result (one-sided Spearman's $\rho$ rank correlation test between $\delta$ and $\alpha, \rho=0.0761$ and $p=0.2004$ for all subjects, and $\rho=-0.0656$ and $p=0.317$ for subjects who are risk averse in both components).

A more general illustration of our main result is shown in Figure 2. Here the relation between $\tau_{u}$ and $\tau_{p}$ is plotted for each subject. The $x$-axis depicts $\tau_{u}$ and the $y$-axis depicts $\tau_{p}$. Small $\tau_{p}$ values correspond to pessimism in probability weighting, and small $\tau_{u}$ values correspond to concave utility. The lower left square represents the largest group of subjects with pessimistic/convex risk attitude. As we can see, no positive correlation between $\tau_{u}$ and $\tau_{p}$ is apparent.

\section{Discussion}

The TO method requires questions to be chained. It is known that this can give subjects incentives to not answer truthfully (Harrison 1986). In theory, subjects could increase their expected payoff by pretending to be more risk averse. Yet, this does not seem to be a practical problem. van de Kuilen and Wakker (2011), who also employed the TO method, found subjects in postexperimental interviews to be unaware of the chained structure, let alone the manipulation possibilities. In our data, we checked for possible manipulation by comparing the reported $x_{i}$ with the possible upper range of $x_{i}$. By our experimental design this ratio equals 0.67 for a risk-neutral agent. ${ }^{8}$ If, however, subjects reported their $x_{i}^{\prime}$ strategically, this ratio would be close to 1 . Our data shows that the mean of the ratios is 0.80 , which is consistent with a reasonable degree of risk aversion.

One may find it surprising that in our study we have mostly convex probability weighting functions, whereas in most previous literature inverse $S$ was prevalent-the more so because we used the same method as Abdellaoui (2000), who found inverse $S$ to be prevailing. We have no conclusive explanation for this difference. But we suspect that it might be related to experimental procedure, subject pool, stake size, and payment method. ${ }^{9}$ Abdellaoui (2000) used small group interviews with subjects who were acquainted with probabilities and expectations, whereas we used group sessions with subjects of diversified backgrounds. He also used a much larger stake size (outcomes between US $\$ 200$ and US $\$ 4,000$ ) but paid only 1 out of 46 subjects on the basis of their decisions, whereas we used outcomes between 1 and 5 euros,

\footnotetext{
${ }^{8}$ For more detailed information, refer to Appendix A.

${ }^{9}$ Unfortunately, we could not use the data of Abdellaoui (2000) for comparison because this data has been lost.
}

and paid all subjects on the basis of their decisions. Another explanation is that the inverse $S$ could not be detected, because the first weight elicited was $\frac{1}{6}$. Our results are not unique though. van de Kuilen (2009) and van de Kuilen and Wakker (2011) found similar results. Thus, the shape of probability weighting is perhaps not as clear as we thought, and more research needs to be done.

For a robust check of our central hypothesis, we also analyzed Bleichrodt and Pinto's (2000) data. Bleichrodt and Pinto (2000) mainly found inverse $S$-shaped probability weighting. We calculated $\tau_{u}$ and $\tau_{p}$ for their data. The one-sided Spearman's $\rho$ rank correlation test between $\tau_{u}$ and $\tau_{p}$ gives qualitatively the same result: $\rho=-0.4328(p=0.999)$. We assumed a power form $u(x)=x^{\alpha}$ for utility functions and $w(p)=\delta p^{\nu} /\left(\delta p^{\nu}+(1-p)^{\nu}\right)$ for probability weighting functions, and we estimated the $\alpha, \delta$, and $\nu$ by minimizing the sum of squared residuals. The one-sided Spearman's $\rho$ rank correlation test between $\alpha$ and $\delta$ gives qualitatively the same result: $\rho=-0.4469(p=$ 0.9993 ). These results suggest that our result is neither limited to our data nor to the shape of the probability weighting function.

\section{Conclusion}

In view of numerous studies exploring risk attitudes, it is now probably less controversial to argue that risk attitudes have two components. Yet to the best of our knowledge, no study has so far addressed the relation between these two components of risk. This question is important because PT would have been a less significant challenge to EU if the curvature of utility and probability weighting had been strongly correlated.

In this paper, we elicited risk attitudes using the trade-off method. We classified utility and probability weighting for each individual subject and analyzed the relation between the two components. We found that, although most individuals exhibit concave utility and convex probability weighting, the two components show no positive correlation. This suggests that the curvature of utility alone is an insufficient proxy for risk attitudes, and that an accurate account of risk attitudes requires the measurement of both.

\section{Acknowledgments}

The authors thank Peter Wakker for his detailed and valuable comments, and Werner Güth, Michel Robe, Rupert Sausgruber, Christoph Vanberg, an anonymous associate editor, and two anonymous reviewers for their helpful comments and suggestions. The authors also thank Han Bleichrodt for sharing some of his experimental data with them. This paper was presented at the 2009 Asia Pacific Meeting of the Economic Science Association in Haifa and the 2009 Spring Meeting of Young Economists in Istanbul. Comments and suggestions of participants are gratefully acknowledged. 


\section{Appendix A. The (Modified) Bisection Choice Procedure}

The detailed algorithm of the (modified) bisection choice procedure is as follows:

1. Given $x_{i}$, we set a range for $x_{i+1}$ 's indifference value. This range should be large enough to include potential indifference values for $x_{i}$, and it should be small enough to allow for a good inference of the indifference point. We used the following equation to determine this potential range:

$$
\begin{gathered}
\underline{x}=\max \left\{0,\left(x_{i}+R\right) \cdot 0.5-r\right\}, \\
\bar{x}=\left(x_{i}+R\right) \cdot 1.5-r .
\end{gathered}
$$

The determination of this range reflects the combined consideration of flexibility and efficiency. Let $x_{m}=(\underline{x}+\bar{x}) / 2$ denote the middle point of the interval $[\underline{x}, \bar{x}]$. Subjects were first presented with a pair of lotteries: $A=\left(x_{i}, 0.5 ; 10\right)$ and $B=\left(x_{i+1}, 0.5 ; 0\right)$, with $x_{i+1}=x_{m}$. To ease calculations only integers were allowed. When $x_{i}$ is not an even integer, the closest even integer larger than $x_{i}$ is taken.

2. If $A$ is preferred, we know that $x_{i+1}$ must be increased to achieve indifference. We thus let $x_{i+1}=\left(x_{m}+\bar{x}\right) / 2$. Likewise, if $B$ is preferred, $x_{i+1}$ must be decreased. We then let $x_{i+1}=\left(x_{m}+\underline{x}\right) / 2$.

3. Repeating this procedure four more times, the interval containing the indifference point will become rather small. Finally, we choose the middle point of the final interval to be $x_{i+1}$.

A drawback of the bisection procedure is that it is not entirely incentive compatible. If subjects are aware of the entire experimental procedure from the start, they may have an incentive to strategically misreport their choices. To see this, note that pretending to be overly risk averse, i.e., choosing $A$ all the time, raises $x_{i+1}$ and thus increases the mean payoff of prospects $B$. Because subjects are paid their preferred prospect in one randomly chosen pair, this misreporting strategy may increase their expected experimental payoff. To make it more difficult to fully grasp the bisection procedure, we added two choices at the beginning elicitation procedure. Therefore, in total, eight choices were taken to elicit each point. The display of these two choices is independent from participant's choices and is expected to make the inference of the whole algorithm more difficult.

The procedure may be best understood with a numerical example. In the experiment, we started the elicitation with the following pair of prospects: $A=(20,0.5 ; 10) \sim B=$ $\left(x_{1}, 0.5 ; 0\right)$. The potential range of $x_{1}$ is $[15,45]$. Participants will then face the sequence of choices shown in Table A.1.

\section{Table A.1 Illustration of the Modified Bisection Choice Procedure}

\begin{tabular}{lccc}
\hline No. & Alternatives & Choice & Inference \\
\hline 1 & $A=(20,0.5 ; 10)$ vs. $B=(30,0.5 ; 0)$ & $A$ & $x_{1} \in[30,45]$ \\
2 & $A=(20,0.5 ; 10)$ vs. $B=(24,0.5 ; 0)$ & $A$ & $x_{1} \in[30,45]$ \\
3 & $A=(20,0.5 ; 10)$ vs. $B=(38,0.5 ; 0)$ & $A$ & $x_{1} \in[38,45]$ \\
4 & $A=(20,0.5 ; 10)$ vs. $B=(34,0.5 ; 0)$ & $A$ & $x_{1} \in[38,45]$ \\
5 & $A=(20,0.5 ; 10)$ vs. $B=(41,0.5 ; 0)$ & $B$ & $x_{1} \in[38,41]$ \\
6 & $A=(20,0.5 ; 10)$ vs. $B=(39,0.5 ; 0)$ & $A$ & $x_{1} \in[39,41]$ \\
7 & $A=(20,0.5 ; 10)$ vs. $B=(40,0.5 ; 0)$ & $A$ & $x_{1} \in[40,41]$ \\
8 & $A=(20,0.5 ; 10)$ vs. $B=(41,0.5 ; 0)$ & $B$ & $x_{1} \in[40,41]$ \\
\hline
\end{tabular}

Based on these choices, $x_{1}$ is set equal to the middle point of the final range $[40,41]$, that is, 40.5 . If subjects choose $A$ all the way, we simply set $x_{1}$ equal to the upper bound of the initial range, which is $45 .{ }^{10}$

Elicitation of probability weights was carried out in a similar manner. For each $p_{i}$, we first presented subjects with a fixed sequence of five pairs of prospects of structure $A=$ $\left(x_{6}, p_{i} ; x_{0}\right)$ and $B=\left(x_{i}, p_{i} ; x_{i}\right)$, where $p_{i}$ is successively set to $0.1,0.9,0.3,0.7,0.5$. Having finished these sequences for all $x_{i}, i=1, \ldots, 5$, we proceeded with the bisection procedure. If there was only one switching point for $p_{i}$, two further iterations would be employed to find the point of indifference. If there were two or more switching points, an interval encompassing all switching points would be determined and a maximum of four iterations of the bisection procedure was employed to find out the indifference probability.

\section{Appendix B. Experimental Instructions}

The experiment was carried out in June 2008 in the computerized laboratory of the Max Planck Institute in Jena. We ran four sessions, with 32 subjects in three sessions and 28 subjects in one session. Each session lasted about 90 minutes. Participants could not observe each other's choices. Rounds for payment were chosen individually, and participants were paid out privately and individually at the end of the experiment.

\section{B.1. General Information}

Thank you for participating in our experiment. Please end all conversations now and switch off your cell phone. Please read the instructions carefully. The money you earn will depend on the choice you make. The money will be paid to you in cash at the end of the experiment. Throughout the experiment, we shall speak of ECU (experimental currency units) rather than euro. The exchange rate between ECU and euro is fixed to $20 \mathrm{ECU}=1$ euro. Please do not communicate during the experiment, and raise your hand if you have questions. We will answer your questions individually. It is very important that you obey these rules, because we would otherwise be forced to exclude you from the experiment and hence from payment.

The experiments consists of four parts. Each part consists of several rounds. In each round you have to make a decision. At the end of the experiment, one round of each part is selected for payment. The sum of these four payments will be your final payment.

\section{B.2. Instructions for the UT Experiment}

The first part of the experiment comprises 42 rounds. In each round, you will be presented with a pair of risky alternatives. Your task is to pick your preferred alternative. To make the comparisons easier, the payoffs are also presented in the upper right corner of the screen. The pairs of risky alternatives will have the following format.

The alternatives shown in Figure B.1 can be better understood by using the following thinking. Imagine a big watch

\footnotetext{
${ }^{10}$ For the current example, one may find eight choices are too much. For later rounds, this will be necessary because $x_{i}$ increases with sequence and so does the potential range of $x_{i}$.
} 


\section{Figure B.1 Illustration of the Lottery Display in the UT Experiment}

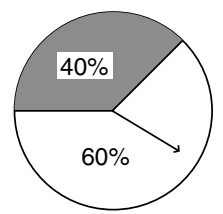

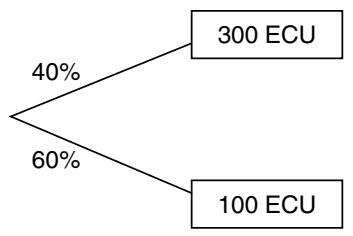

Alternative $A$

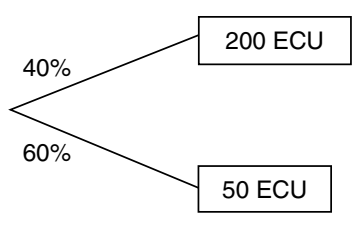

Alternative $B$ with one arm. In the figure, $40 \%$ of the panel is covered by black and $60 \%$ of the panel is covered by white. The arm of the watch stops equally likely at each position of the watch. Suppose now you have chosen alternative $A$ from the above pair. Then, if the arm stops in the black area, you are paid 300 ECU, if the arm stops in the white area, you are paid 100 ECU. (Equivalently, had you chosen $B$ you would be paid $200 \mathrm{ECU}$ in case of black and $50 \mathrm{ECU}$ in case of white.)

At the end of this part of the experiment, one of your choices will be randomly selected and played, and the resulting outcome will be your experimental earning in this part.

\section{B.3. Instructions for the PW Experiment}

This part is similar to the first part. Again you will be asked for your preference between two lotteries, the difference being that lottery $B$ always gives a fixed payoff. Another difference is that the probabilities in lottery $A$ change for each decision. Using the picture of the first part, the division of the circle between black and white changes for each decision. Please think carefully before each decision, because a confirmed choice cannot be changed.

\section{References}

Abdellaoui, M. 2000. Parameter-free elicitation of utility and probability weighting functions. Management Sci. 46(11) 1497-1512.

Abdellaoui, M., F. Vossmann, M. Weber. 2005. Choice-based elicitation and decomposition of decision weights for gains and losses under uncertainty. Management Sci. 51(9) 1384-1399.

Bleichrodt, H., J. L. Pinto. 2000. A parameter-free elicitation of the probability weighting function in medical decision analysis. Management Sci. 46(11) 1485-1496.

Camerer, C. F. 1989. An experimental test of several generalized utility theories. J. Risk Uncertainty 2(1) 61-104.

Fischbacher, U. 2007. z-Tree: Zurich toolbox for ready-made economic experiments. Experiment. Econom. 10(2) 171-178.

Goldstein, W. M., H. J. Einhorn. 1987. Expression theory and the preference reversal phenomena. Psych. Rev. 94(2) 236-254.

Greiner, B. 2004. An online recruitment system for economic experiments. K. Kremer, V. Macho, eds. Forschung und Wissenschaftliches Rechnen. GWDG Bericht 63, Ges. für Wiss. Datenverarbeitung, Göttingen, 79-93.

Harrison, G. W. 1986. Risk aversion and preference distortion in deterministic bargaining experiments. Econom. Lett. 22(2-3) 191-196.

Harrison, G. W., E. E. Rutström. 2008. Risk aversion in the laboratory. J. C. Cox, G. W. Harrison, eds. Risk Aversion in Experiments, Vol. 12. Research in Experimental Economics. Emerald Group Publishing Limited, Bingley, UK, 41-196.

Kahneman, D., A. Tversky. 1979. Prospect theory: An analysis of decision under risk. Econometrica 47(2) 263-292.

Qiu, J., E.-M. Steiger. 2010. On the impact of risk attitudes on belief updating: An experimental study. Working paper, Strategic Interaction Group, Max Planck Institute of Economics, Jena, Germany.

Starmer, C., R. Sugden. 1989. Violations of the independence axiom in common ratio problems: An experimental test of some competing hypotheses. Ann. Oper. Res. 19(1) 79-102.

van de Kuilen, G. 2009. Subjective probability weighting and the discovered preference hypothesis. Theory Decision 67(1) 1-22.

van de Kuilen, G., P. P. Wakker. 2011. The midweight method to measure attitudes toward risk and ambiguity. Management Sci. Forthcoming.

Wakker, P. 1994. Separating marginal utility and probabilistic risk aversion. Theory Decision 36(2) 1-44.

Wakker, P., D. Deneffe. 1996. Eliciting von Neumann-Morgenstern utilities when probabilities are distorted or unknown. Management Sci. 42(8) 1131-1150. 